\title{
Under-sampling in PET scanners as a source of image blurring
}

\author{
C.J. Thompson*, S.St. James, N. Tomic \\ Montreal Neurological Institute and Medical Physics Unit, McGill University, Montreal, Que., Canada \\ Received 9 November 2004; received in revised form 14 January 2005; accepted 20 January 2005
}

Available online 28 March 2005

\begin{abstract}
The spatial resolution of PET scanners is degraded by a number of causes, both fundamental to the nature of positron decay, and to detectors. The crystal dimensions, their placement in the block and readout all contribute to the loss of resolution. We investigated the relative effects of sampling of the image space by the detectors, and the use of block detectors in a whole body PET scanner on resolution degradation. Three sources were mounted on a linear translation stage which moved them trans-axially through the central field of a Siemens CTI HR + PET scanner. A 140 frame study was acquired as the sources moved horizontally $0.5 \mathrm{~mm}$ between frames. The vertical projection from each frame was summed over all slices. The FWHM of each source's representation was estimated in each frame in the summed projections. The response functions were much sharper from crystals at the edge of the detector blocks, than from those from the central crystals. They were consistent with the effects of source size, non-collinearity, and crystal dimensions. The response from the central crystals were degraded by an additional term of $1.2 \mathrm{~mm}$ added in quadrature to the other blurring effects. The intensity of the image of a small source depended on the source location, as did the FWHM of the response of the central crystals in the block detectors. The response functions of edge crystals were found to be sharper than those of the central crystals in the block detectors. However, a more interesting finding is that when very small sources are imaged in PET scanners their apparent intensity and their associated response functions depend on their location along any projection. This under-sampling results in resolution loss equivalent to about $\frac{1}{2}$ of the crystal width.
\end{abstract}

(C) 2005 Elsevier B.V. All rights reserved.

PACS: 87.59.V; 87.59; 87.62

Keywords: PET; Spatial resolution; Under-sampling; Block detectors

\footnotetext{
*Corresponding author. Montreal Neurological Institute \#798, 3801 University St. Montreal, Que., Canada H3A 2B4. Tel.: + 15143988505 ; fax: + 15143988507.

E-mail address: christopher.thompson@mcgill.ca (C.J. Thompson).
}

\section{Introduction}

Spatial resolution and image noise are key factors in all instruments used in Nuclear Medicine. In PET the fundamental nature of positron 
decay sets limits on spatial resolution which are absent when imaging isotopes which emit $\gamma$-rays. The positron emitted during the decay of the parent isotope shares the energy associated with the mass difference between parent and daughter nuclei, and must lose this energy before combining with an electron and annihilating. The positronrange blurring affects all PET scanners and is greater when imaging tracers labelled with ${ }^{11} \mathrm{C}$ and ${ }^{15} \mathrm{O}$ than with ${ }^{18} \mathrm{~F}$ [1]. The kinetic energy of the positron-electron pair at the time of annihilation causes the pair of $\gamma$-rays to appear to travel in not quite opposite directions. This angular deviation of about $0.5^{\circ}$ [2] blurs PET images to a greater extent in human scanners than it does in those designed for small animal imaging.

The size of the crystals used to detect the $511 \mathrm{keV} \gamma$-rays has been the main instrumental cause of the resolution loss in PET scanners. The ideal geometrical response of two crystals in coincidence to a pair of perfectly collinear $\gamma$-rays would be a triangle if the source was mid-way between the crystals. This degrades to a trapezoid if the source is nearer one detector, which prompted us to examine the blurring effects in the centre of the scanner where geometrical effects are minimized.

Currently almost all PET scanners use "block detectors" [3]. These are made either by cutting a solid piece of scintillator part-way through to various depths and coupling this to four photomultipliers, or by coupling individual crystals to a glass light guide which has a similar pattern of cuts. Either technique allows for much smaller crystals than would be economically feasible with 1:1 coupling.

In their study of the limits for spatial resolution for PET scanners, Derenzo et al. [4] proposed a formula to determine the spatial resolution of PET scanners, viz:

$\Gamma=1.25 \sqrt{(c / 2)^{2}+(0.0022 D)^{2}+s^{2}+b^{2}}$

where $\Gamma$ is the spatial resolution, $c$ is the crystal width, $D$ is the diameter of the detector ring, $s$ is the source size (which includes the physical size and positron range blurring in tissue), and $b$ is an additional term which is required to explain the poorer resolution of scanners with block detectors. The factor 1.25 represents the interpolation and back-projection in the image reconstruction, and $0.0022=\left(\frac{1}{2}\right) \tan \left(0.25^{\circ}\right)$. This is based on an examination of 17 PET scanners whose performance data had been published in 1993. This paper showed that if the effects of source size and ring diameter are removed this equation is reduced to a linear one such that the resolution is a linear function of crystal width. In scanners with 1:1 coupling, the line goes through the origin, but it is offset in scanners with block detectors. Later Lecomte [5] proposed that for scanners with 1:1 coupling the term " $b$ " is zero, but when the crystals were electronically multiplexed $b \sim 1.2 \mathrm{~mm}$ and when light-sharing is used $b \sim 2.4 \mathrm{~mm}$.

We previously investigated the blurring due to Compton scattering of the primary $\gamma$-rays within detector blocks and narrow crystals [6], using the Monte-Carlo simulation program PETSIM [7] and found that this has a minimal impact on the fullwidth at half-maximum (FWHM) of the coincidence aperture function (CAF), although it does effect the full-width at tenth maximum (FWTM) since the scattered ray interacts with the crystal some distance away from the point of scattering. It thus seems unlikely that the blurring assumed to be related to the block effect could be accounted for by physics of interaction of $\gamma$-rays with dense crystals.

Recently we obtained a block detector of the type used in the CTI ECAT HR + PET scanner, and used a point-like source and a thin crystal to ascertain if the blurring attributed to block detectors was due to the inherent properties of the detector. Our findings show that there is no blurring in response from the edge crystals, but that there was blurring equivalent to $b=1.2 \mathrm{~mm}$ for the central crystals [9].

Prior to the introduction of block detectors, most PET scanners had two modes of operation, one in which the detector array remained stationary, and another in which the detector array moved in some way. This was done to increase the spatial sampling. The two most common methods were a continuous rotary motion [10] of the entire detector array with an orbital radius of the order of the crystal width commonly known as 
"wobbling". Another was called dichotomic [11] first, and later "clamshell" motion in which the array was made in two halves which were hinged at the bottom, and could open up to the width of one crystal [12]. The rationale for these sampling schemes is that the lines of response (LOR) joining the centres of stationary crystals on a ring do not adequately sample the image space.

In the studies by Derenzo [4] and Lecompte [5] the scanners with 1:1 crystal coupling that are determined to have no measurable block effect are also scanners that used clam-shell sampling. Most of the evaluated scanners with block detectors used stationary detectors.

In a paper by our group on the performance of the Scanditronix PC2048B scanner [13] we reported a FWHM in the centre of the field of view (FOV) of $4.6 \mathrm{~mm}$ when the detectors wobbled, and $5.9 \mathrm{~mm}$ when the detectors were stationary. This scanner is interesting in the current context since it was one of the few PET scanners which had block detectors and which offered the option of wobbled acquisitions to provide higher resolution. If one inserts the crystal width and ring diameters into Eq. (1), the apparent "block effect" would be reduced by $1.7 \mathrm{~mm}$ to $0.7 \mathrm{~mm}$ in the wobbled mode. This suggests that under-sampling may be a substantial cause of image blurring in PET scanners with block detectors.

Further evidence of the existence of undersampling comes from the document [14] from the National Electric Manufacturer's Association (NEMA) on the measurement of the performance of PET scanners. In the section on the measurement of the spatial resolution of PET scanners, the document requires that the measurement representing the central FOV be made at $1 \mathrm{~cm}$ from the actual centre "to represent the centre of the FOV, but positioned to avoid any inconsistent results associated with the very centre of the FOV'. This is because if a source is placed at the very centre of a PET scanner, it is also at the exact centre of the response function of a pair of detectors in every projection. In this special case there is no possible blurring associated with under-sampling. The inconsistencies referred to in the NEMA document are well illustrated in a report on the performance of the Concorde Microsystems
MicroPET R4 [15]. In this scanner, the FWHM changes rapidly from $1.65 \mathrm{~mm}$ in the exact centre of the FOV to greater than $2.5 \mathrm{~mm}$ at a distance of $1 \mathrm{~cm}$ from the centre.

The purpose of the present paper is to elucidate the effects of under-sampling in a widely used PET scanner, after determining that the blurring reported in the performance evaluation of this instrument could not be attributed to the properties of the block detectors. The observations made here relate to one scanner. This scanner has slightly different crystal widths of the central and edge crystals of the block. We have taken care to take this into account, in the expectations that our findings would be more generally applicable to any scanner using conventional stationary block detectors.

\section{Materials and methods}

The measurements were made on a SiemensCTI ECAT Exact HR + (CPS Innovations, Knoxville TN) scanner [8] installed in the Montreal Neurological Institute's McConnell Brain Imaging Centre since 1997. The scanner uses 288 blocks (each of which is an $8 \times 8$ crystal array) mounted on four rings of $82.5 \mathrm{~cm}$ in diameter. There are thus 576 crystals around the circumference of a circle. The 2D sinogram files from this scanner are formatted as LOR in which alternate values come from crystal pairs which are even and odd numbers of crystals apart. These lines are therefore not strictly parallel, as illustrated in Fig. 1. The purpose of this is to double the number of linear samples at the expense of halving the number of angular samples from 288 to 144 . The central crystals in these detector blocks were previously measured [9] to be $4.4 \mathrm{~mm}$ wide with a $1 \mathrm{~mm}$ saw cut, and the edge crystals are $4.2 \mathrm{~mm}$ wide (to allow for the light shielding metal encapsulation of the block). Since the crystal separation is $4.5 \mathrm{~mm}$, the LOR are separated by $2.25 \mathrm{~mm}$, yielding a sampling frequency of 4.44 samples/cm near the centre of the FOV.

If one considers only the strictly geometric effects, (i.e. ignoring positron range and noncollinearity) the response of a pair of crystals to a 


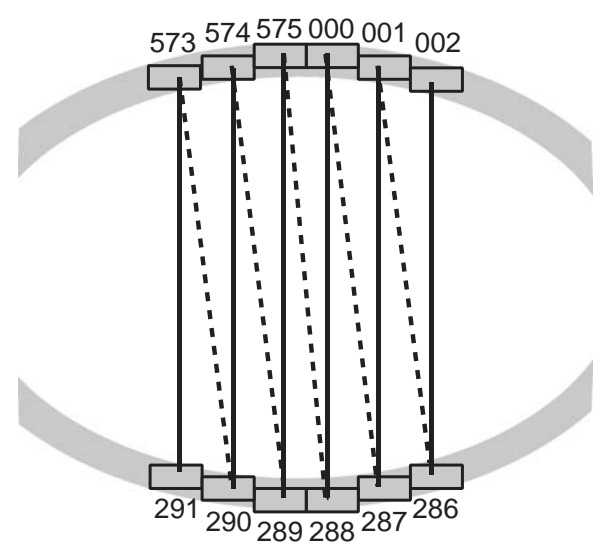

Fig. 1. The lines of response which form one projection are interleaved from two adjacent angles in order to double the spatial sampling. The error resulting from this approximation is very much smaller than illustrated here when the crystals are narrow, and only the central region is considered. In this vertical projection the solid lines (joining even numbered crystals) are truly vertical, whereas, the dashed lines are at an angle $(360 / 576)$ or $0.625^{\circ}$

point source anywhere in the space separating them can be modelled as the probability of an arbitrary line through that source intersecting the front face of both crystals. If one considers only events in a plane joining two crystals whose width is $C$, and whose separation is $2 R$, then, as illustrated in Fig. 2, the response for a point $x, y$ is zero if $|x|>C / 2$. If $|x|<C / 2$ the line through $x, y$ will intersect both crystals if the angle which the $\gamma$ rays make with the line joining the two crystals is between $\alpha$ and $\beta$ where these angles are defined as

$\alpha=\tan ^{-1} \frac{(C / 2-|x|)}{(R+|y|)}, \quad \beta=\tan ^{-1} \frac{(C / 2+|x|)}{(R+|y|)}$

or

$\alpha=\tan ^{-1} \frac{(C / 2-|x|)}{(R+|y|)}, \quad \beta=\tan ^{-1} \frac{(C / 2-|x|)}{(R-|y|)}$

depending on whether the point $(x, y)$ is inside or outside the dashed lines joining the opposite extremities of the crystals in Fig. 2. The response of a pair of crystals of which one is laterally offset can be derived by skewing these angles by a

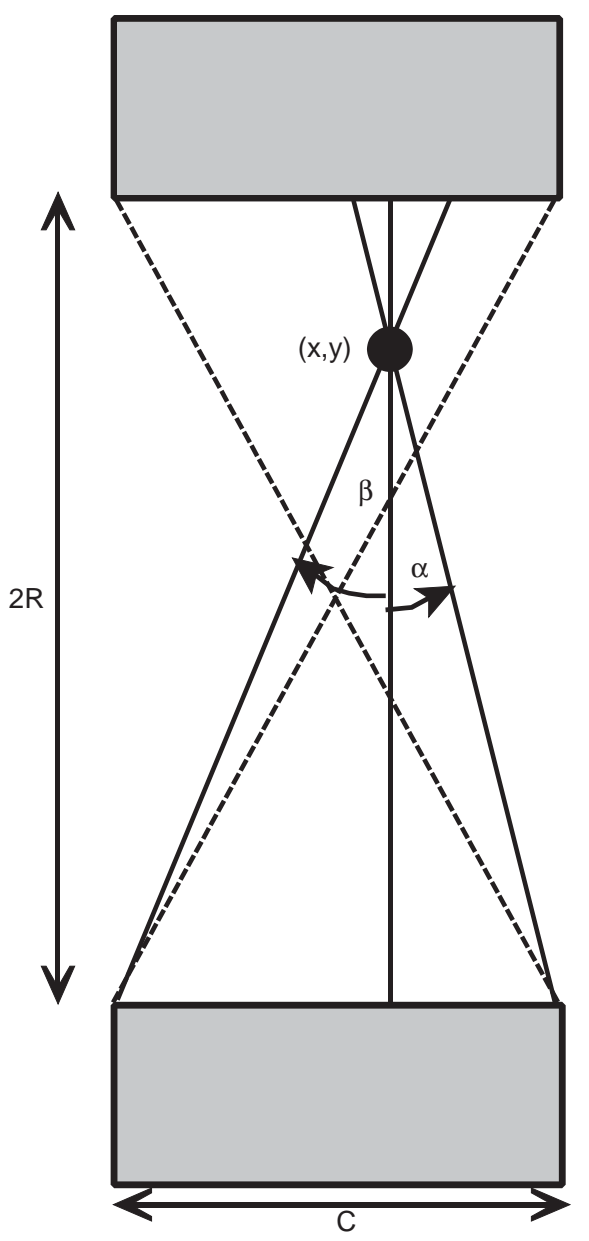

Fig. 2. A positron emitting source at $(x, y)$ in the space between two crystals of width $=C$, and separation $=2 R$, has a probability of both $\gamma$-rays intersecting the crystals which depends on its position being within, or beyond the dashed lines joining the crystals' opposite corners.

horizontal translation of the lower crystal by the crystal width, $C$.

A program was written in MATLAB (The Mathworks Inc., Natick MA) to map out the response of pairs of crystals like those illustrated in Fig. 1 as a function of the space between them and the crystal width. At each point, $(x, y)$ the appropriate values of $\alpha$ and $\beta$ from Eqs. (2) or (3) were used as limits of integration to determine the number of counts detected from a source in that position. 
A wooden support was constructed to mount two rod sources ${ }^{68} \mathrm{Ge}$ of the type used to perform attenuation correction on this scanner, and a point-like ${ }^{22} \mathrm{Na}$ source (Isotope Products Laboratories, Valencia CA) with inter-source separations of $15 \mathrm{~mm}$. This support was attached to a linear translation stage (Compumotor Inc., Dorhorf Park CA). The translation stage was placed on the scanner's couch. The sources were placed axially in the scanner with one of them at the geometrical centre of the FOV using the patientpositioning lasers. The translation stage was programmed to move in $0.5 \mathrm{~mm}$ increments when requested.

A 140-frame dynamic scan was initiated, with a frame time of $60 \mathrm{~s}$ and a delay of $10 \mathrm{~s}$ between frames. During this delay between frames, the sources were moved horizontally by $0.5 \mathrm{~mm}$. The study protocol was for a $2 \mathrm{D}$ acquisition with the septa extended. Angular compression (mashing) was disabled to provide the maximum linear sampling in the sinograms. On-line randoms subtraction was performed to reduce the file size. The randoms rate was less than $5 \%$ of the true counts in the experiments.

In this acquisition mode each frame of the sinogram requires 100 Mbytes of disk space. The resulting 1.4 Gbyte sinogram file was copied to a VAX-STATION 4600 (Digital Equipment Corporation, Maynard, MA). A program was written to extract only the vertical projection from all 140 frames and sum the projection over all 63 slices. The FWHM of the response to each source in each frame was measured by locating each of the three peaks, and interpolating between the sample values just above and below the half-way point to measure the FWHM according the procedure suggested in the NEMA reference document [14]. The FWHM was not measured by fitting the response to a Gaussian. Three distinct FWHM values selected for further study were the best FWHM values (corresponding to each source $=s$ response from the edge crystals in the blocks), the average values from crystals within the blocks when the source was in the best alignment (with respect to the crystal-pair $=s$ response function), and the average values from crystals within the blocks when the source was in the worst alignment.
The 140 summed projections were converted to text file and imported into MATLAB for display as surface plot (see Figs. 5 and 6). The horizontal axis of these plots is the frame number or position of the sources in units of $0.5 \mathrm{~mm}$. The axis running into the page is the sample number along the summed projection in units of $2.25 \mathrm{~mm}$. The vertical axis is proportional to the number of counts acquired in all planes in $60 \mathrm{~s}$. No crystal efficiency normalization was applied as it would be during image reconstruction in order to demonstrate the relative sensitivity of each crystal pair.

\section{Results}

The geometric response (ignoring positron range and non-collinearity blurring) to a uniform positron emitting source from a set of detector pairs is illustrated in Fig. 3a. The gaps in the central region illustrate the necessity of introducing additional samples in the sinogram. When the response of the LORs which are skewed by one crystal are added to the truly vertical lines, as in Fig. $3 b$, the image space sampling is improved, but it is not complete.

A gray-scale image representing the detectorpairs' response to the three sources as they move through the scanner is shown in Fig. 4. The intensity of the points on the three lines represents the relative count-rates of crystal pairs in the detector blocks. The pixels represent a displacement of the sources (along the vertical axis) of $0.5 \mathrm{~mm}$ and the separation of the LOR from adjacent LORs (along the horizontal axis) of $2.25 \mathrm{~mm}$. The two sets of arrows show the coordinates from which the plots in Figs. 9 and 10 were derived.

The response function is portrayed as a MATLAB surface plot in Fig. 5. This figure has the appearance of three ridges of mountains. The peaks and valleys correspond to source being lined up with the centres of two crystals, and gaps between crystals in opposing blocks. The region of the response to only one of the sources is selected in Fig. 6 by segmenting the data, and rotating the axes of the surface plot. The individual peaks and valleys are more visible in the view, as is the relative efficiency of the central crystals in the 

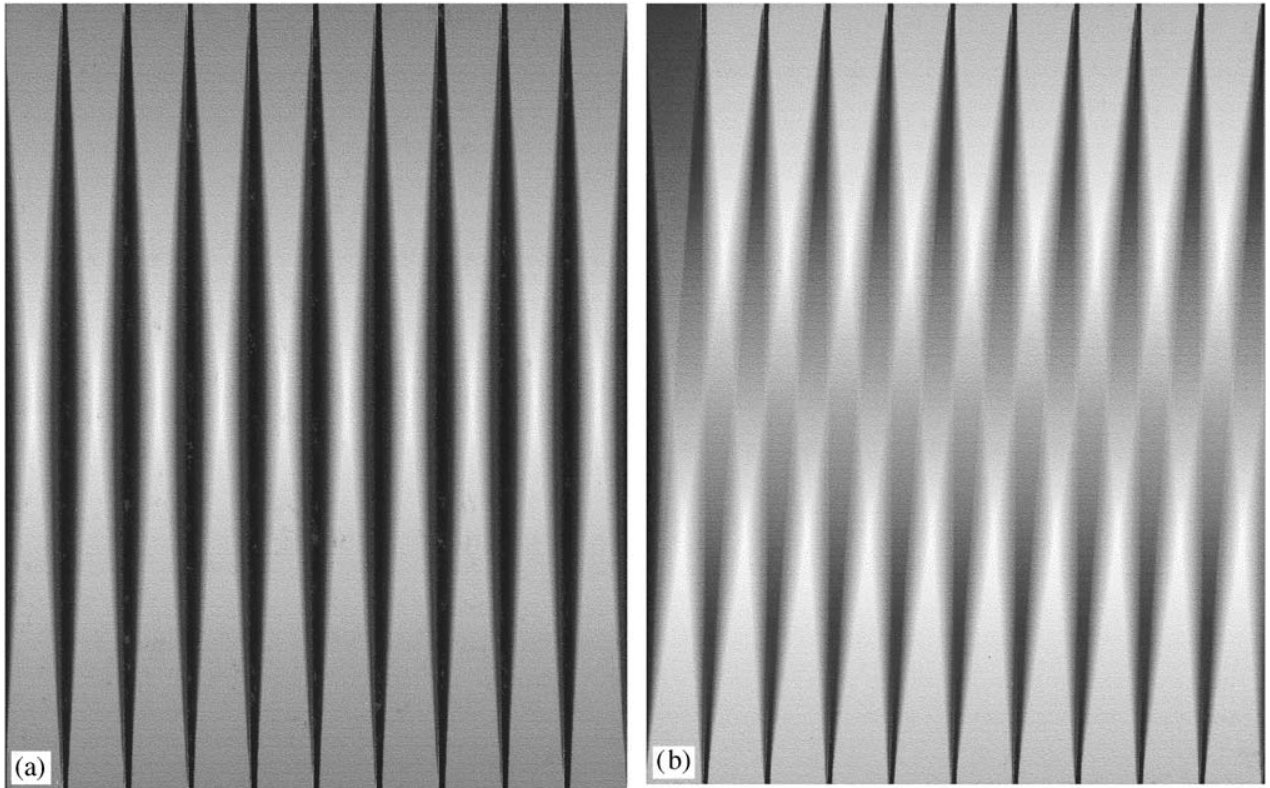

Fig. 3. The geometric coincidence response function from pairs of equally spaced crystals is shown in the left image. If the response from pairs of crystals which are offset by one element is added and interleaved, the total response is more uniform, but the undersampling is still visible.

block compared with the outer ones (which appear as deeper valleys, and lower peaks).

The CAFs of three crystal pairs are illustrated in Fig. 7. These CAFs are sampled at $2.25 \mathrm{~mm}$. When the source is positioned between two outer crystals, the FWHM was measured as $3.2 \mathrm{~mm}$. The worst case is when the source is placed midway between the LORs which yields FWHMs as high as $5.7 \mathrm{~mm}$. Average values from central crystal pairs are $4.98 \mathrm{~mm}$. In Fig. 8, a horizontal row and a vertical column (extracted from Fig. 3) demonstrate the effect of sampling density on the observed detector response function. The response is very consistent for the finely sampled vertical axis (where the source is moving in $0.5 \mathrm{~mm}$ increments between the detectors) and more erratic on the horizontal axis where the sampling is $2.25 \mathrm{~mm}$ due the inter-crystal spacing.

The FWHM of each crystal-pair's response to each of the three sources is shown in Fig. 9. Each of the three sources is represented by a different line style. The average FWHM for the three sources is $4.98 \mathrm{~mm}$. The best resolution is obtained from the crystal pairs at the edge of the blocks (the curves which dip to about $3 \mathrm{~mm}$ ). One section of the FWHM response is shown enlarged in Fig. 10. The fluctuations in FWHM are seen to range from 5.8 when the source is mid-way between two crystals near the centre of a block, to $2.92 \mathrm{~mm}$ when the source is centred on two edge crystals. The results from various crystal-pair combinations are summarized in Table 1. The average FWHM with the source well aligned between two crystals is $0.9 \mathrm{~mm}$ better than when it is mid-way between two LORs.

\section{Discussion}

Normally the performance of PET scanners is assessed from the reconstructed images whereas the performance of detectors is assessed by moving a very small source between them. In this work we have moved a small source across the FOV of a complete scanner during a multi-frame study. This approach attempts to capture the traditional scanner and bench measurements. We chose not to present the data in the form of reconstructed 


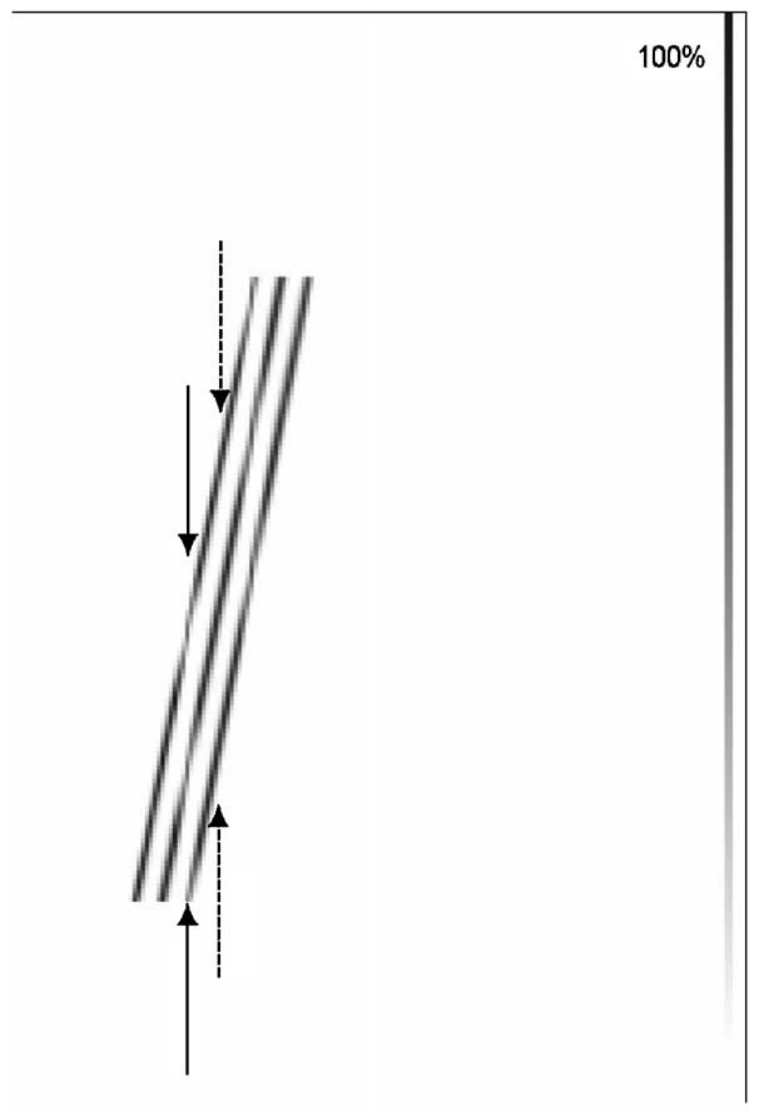

Fig. 4. Image of the detector pairs' response to the three sources as they move across the scanner. Each horizontal row in this figure represents a central region from the vertical projection extracted from one sinogram frame. The solid and dashed lines indicate, respectively, from where the vertical projections shown in Figs. 9 and 10 were derived.

images in order to distinguish between the response of the central and edge crystals in the blocks. This demonstrated a significant change in FWHM between the edge crystal CAFs and those from the central crystals. Since there are fewer LORs between exclusively edge crystals, and the edge crystals are less efficient (the deep valleys in Fig. 7), there are more LORs which blur the image than those to improve it.

Fig. 8 and 9 show that the FWHM changes by over $1 \mathrm{~mm}$ when the source is moved from the centre of a CAF to between two adjacent ones. However, this is obtained from one projection. In the formation of the image, all projections are used, and so a point source will appear well resolved in some projections and poorly resolved in others. This may account for the factor 1.25 in Eq. (1). This has been attributed to the inevitable interpolation due to reconstruction. However, in a scanner with wobbled detectors the sampling is much finer, allowing for better and more consistent spatial resolution.

The data plotted in Fig. 9 clearly demonstrates the narrower response from edge crystals, whereas the data plotted in Fig. 6 show the reduction in efficiency in LORs from edge crystals. The edge crystals of this block are thinner than the others in the block (4.2 vs. $4.4 \mathrm{~mm})$ The reduction in efficiency at the crystal edges is probably due to the reduced probability of multiple interactions of a $\gamma$-ray within the crystal depositing enough energy to be detected. This is seen in Monte Carlo simulations. It could probably be demonstrated by repeating the experiment with different lowenergy discrimination settings. The narrower response functions in the edge crystals in the detectors used in this study is partly due the fact that they are physically smaller than the central crystals. However, in all block detectors there is a decreased probability of multiple interactions in the edge crystals being detected in the photo-peak.

The observations made here relate to one scanner. This scanner has slightly different crystal widths of the central and edge crystals of the block. We have taken care to take this into account, in the expectations that our findings would be more generally applicable to any scanner. The manufacturer presumably used narrower crystals at the edges of the blocks in order to keep the sampling interval constant, while employing light-tight detector blocks.

Our results demonstrate two components of blurring, one due to under-sampling (which causes the FWHM for the central crystals to fluctuate between 5.45 and $4.55 \mathrm{~mm}$ ), and another due the variation between central and edge crystals (which causes the FWHM to improve from 4.55 to below $3 \mathrm{~mm}$ for the edge crystals). One could thus expect some improvement in scanner performance if the spatial sampling were increased. However, it seems unlikely that any manufacturer would want 


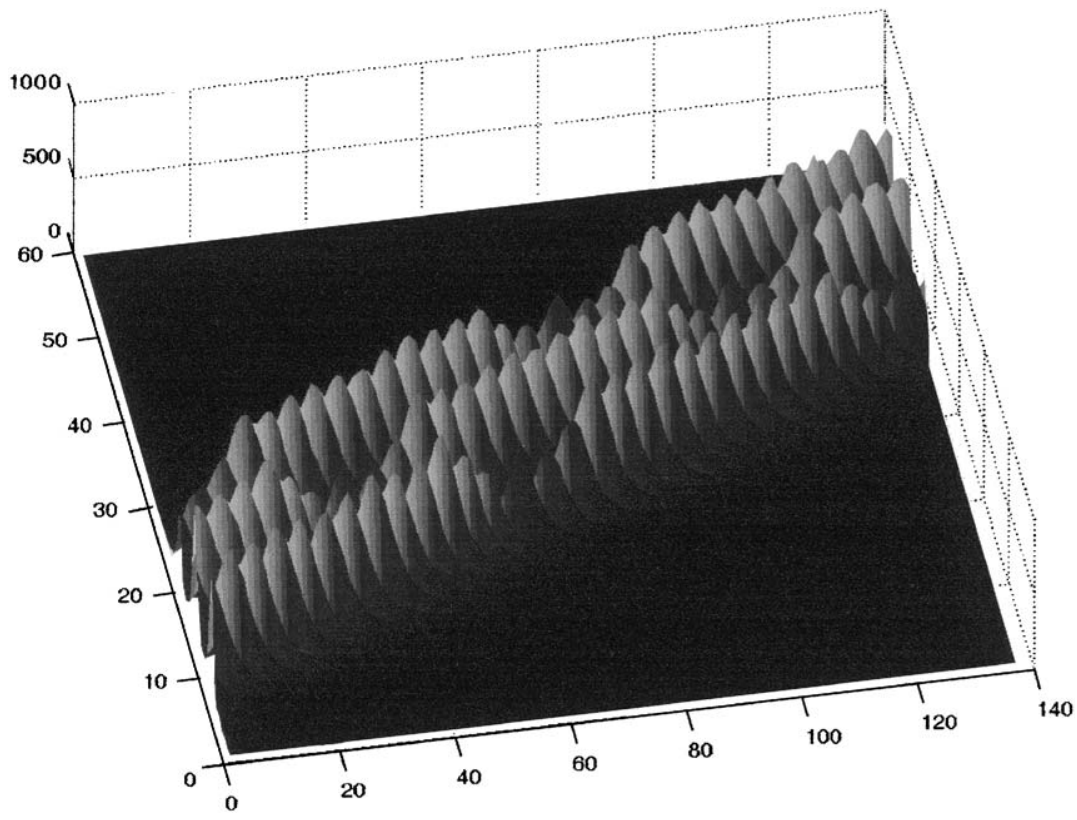

Fig. 5. Surface plot of the data experimental results. The horizontal axis is the frame number, the axis going into the page is the relative sample number along the projection, and the vertical axis the relative number of counts. The three diagonal ridges represent the detector pairs' responses to the three sources, with the lower regions being the edge crystals' responses, and the higher points being the blocks' central crystals' responses.

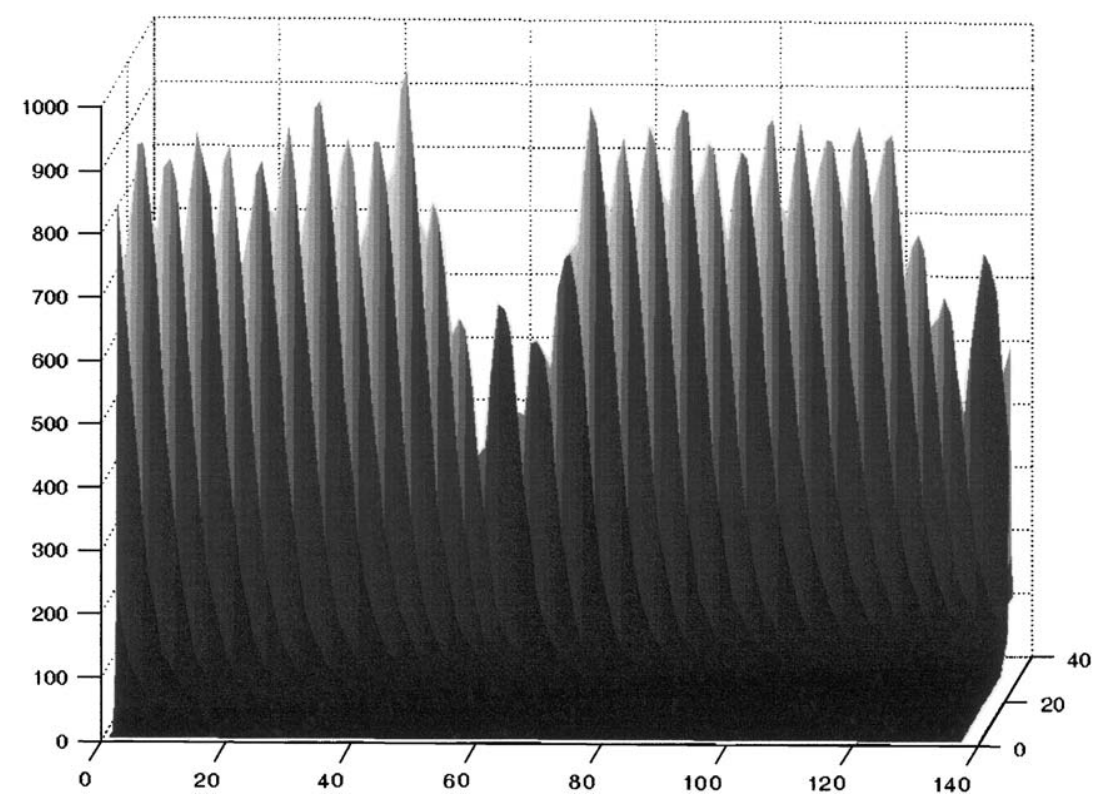

Fig. 6. Surface plot of the response to one source only. The horizontal axis is the frame number, the axis going into the page is the relative sample number along the projection, and the vertical axis the relative number of counts. The deep central valley is response at the edges of two adjacent blocks. 


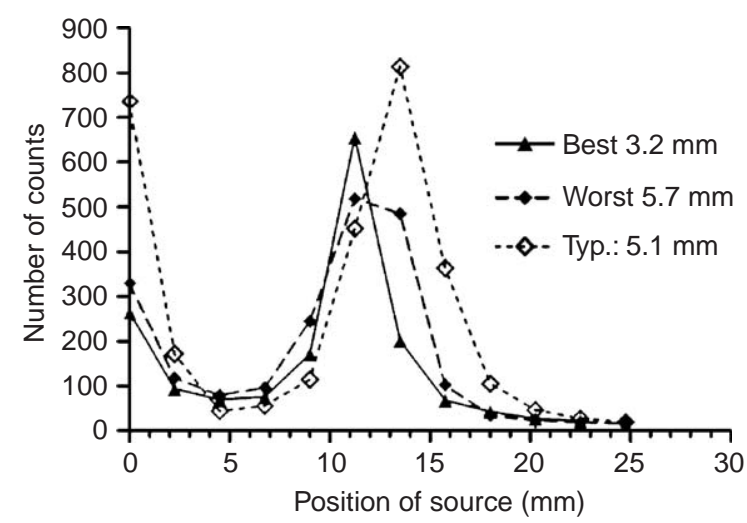

Fig. 7. Raw summed projection data illustrating the range of response functions from different detector pairs. The solid line shows the response of crystal pairs at the edge of two blocks with a FWHM of $3.2 \mathrm{~mm}$. The dashed line is the response near the centre of the blocks at a point where the source was between two LORs. The dotted line is from a typical pair of crystals near the centre of the block. The sampling interval is $2.25 \mathrm{~mm}$.



Fig. 8. The response function extracted from sinograms from crystals at the edge of blocks. The vertical response line is sampled every $0.5 \mathrm{~mm}$, and the horizontal response line is sampled every $2.25 \mathrm{~mm}$.

to reintroduce wobbling in whole body PET scanners.

A form of wobbling may be a realistic proposition for a small animal PET scanner. These instruments have much smaller detectors, and it would be possible to wobble the bed, rather than the detectors (1-2 $\mathrm{mm}$ diameter) in a manner which would be almost imperceptible during the

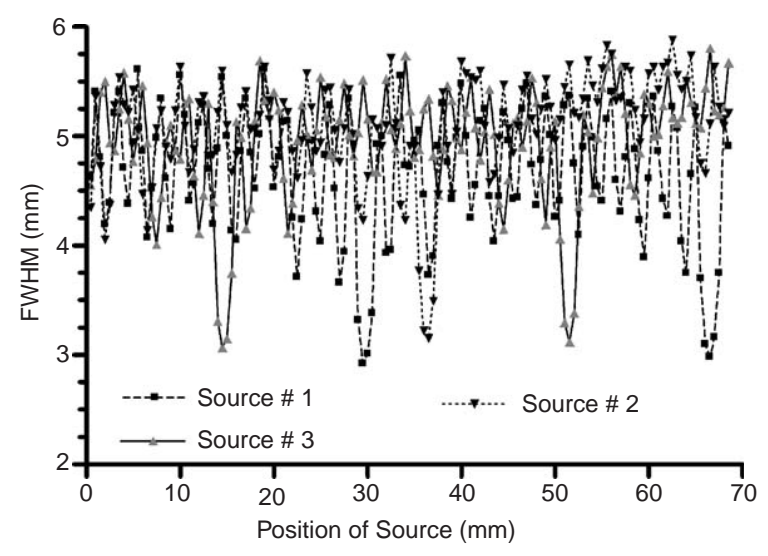

Fig. 9. The FWHM is plotted for all three sources for the entire $70 \mathrm{~mm}$ of source movement.

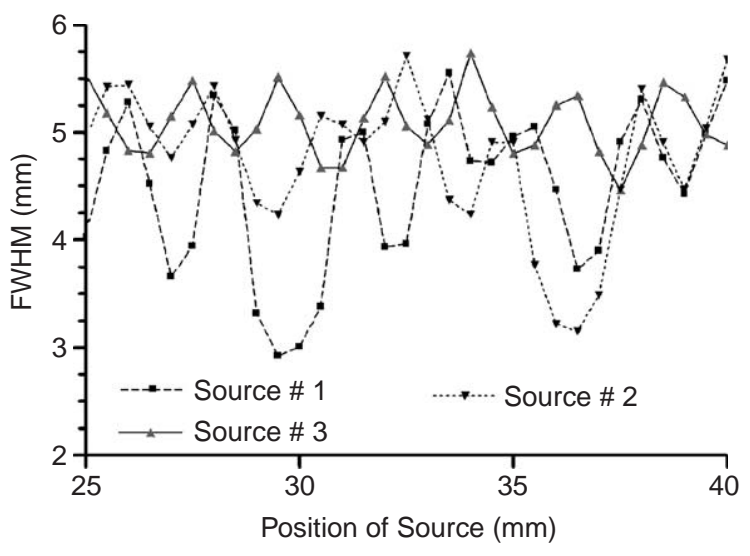

Fig. 10. Expanded version of FWHM plot vs. source position in the region where two sources crossed block boundaries as evidenced by the improvement on FWHM near positions 30 and 36 .

Table 1

FWHM in $\mathrm{mm}$ of the response function of different crystals in a block showing the best, worst and average values obtained when the source is well aligned or mis-aligned with the centreline of the crystals

\begin{tabular}{lllll}
\hline & $\begin{array}{l}\text { Edge } \\
\text { best }\end{array}$ & $\begin{array}{l}\text { Central } \\
\text { best }\end{array}$ & $\begin{array}{l}\text { Central } \\
\text { worst }\end{array}$ & $\begin{array}{l}\text { Ave. of } \\
\text { all central }\end{array}$ \\
\hline Source 1 & 2.92 & $4.20 \pm 0.26$ & $5.33 \pm 0.16$ & $4.76 \pm 0.48$ \\
Source 2 & 3.07 & $4.70 \pm 0.36$ & $5.49 \pm 0.17$ & $5.03 \pm 0.40$ \\
Source 3 & 3.15 & $4.76 \pm 0.34$ & $5.54 \pm 0.18$ & $5.14 \pm 0.39$ \\
Average & 3.05 & 4.55 & 5.45 & 4.98 \\
\hline
\end{tabular}


scan, but could improve the sampling. This has already been tested using a discrete bed motion by Chatziioannou et al. [16]. If continuous motion is used, one can make the sampling uniform in all projections, thus eliminating interpolation.

Another way to improve the sampling is to use dual layered detectors. These are been used to improve the radial blurring due to crystal penetration in the CPS HRRT [17] scanner. However, in that scanner both layers crystals were radially aligned. In our first instrument designed for positron emission mammography (PEM) we cut a crystal block from both sides and offset the saw between sides [18], so that the centres of the crystals in each layer were offset by $\frac{1}{2}$ of the crystal width. Although this makes the crystals more complex to manufacture, it is much simpler than the mechanical system needed to wobble the whole detector array. This may be an option for a human PET scanner.

\section{Conclusions}

This study has demonstrated that there appear to be two components which cause excess blurring in PET images, one due to the coupling of multiple crystals on fewer PMTs and another due to undersampling. Both of these effects serve to blur PET images acquired by a human whole body scanner. The effects are similar in their magnitude. Block detectors are much less expensive to manufacture than those with individual light sensors on each crystal. Whole body PET scanners would not be cost effective without them. We have demonstrated that some of the blurring due to block detectors would be reduced by increasing the sampling. Perhaps this could be achieved in small animal scanners by wobbling the bed, and in human-scale PET scanners by the use of dual layer detectors in which the layers are offset. Further work is required to investigate the utility and cost/benefit of these options to improve the spatial resolution of PET scanners.

\section{Acknowledgements}

This work was funded by an operating grant (to CJT) from the Natural Science and Engineering
Council of Canada: 36672-02. The PET facility at the Montreal Neurological Institute is supported by a Group grant from the Canadian Institutes for Health Research: MCG 13972. The help of Samuel Richard with the source holder and some aspects of the data analysis are gratefully acknowledged.

\section{References}

[1] S.E. Derenzo, IEEE Trans. Nucl. Sci. NS-33 (1986) 565.

[2] P. Colimbino, B. Fiscella, L. Trossi, Nuovo Cimento 38 (1965) 707.

[3] M.E. Casey, R. Nutt, IEEE Trans. Med. Sci. 33 (1986) 460 M.E. Casey, R. Nutt, T. Douglas, US Patent, 4,749,863, 1988.

[4] S.E. Derenzo, W.W. Moses, R.H. Huesman, T.F. Budinger, in: K. Uemura, N.A. Larsen, T. Jones, I. Kanno (Eds.), Quantification of Brain Function, vol. 25, Elsevier, Amsterdam, The Netherlands, 1993.

[5] R. Lecomte, Université de Sherbrooke, Canada, Que., personal communication, 1995.

[6] C.J. Thompson, IEEE Trans. Nucl. Sci. NS-37 (1990) 718.

[7] C.J. Thompson, J.J. Moreno-Cantú, Y. Picard, Phys. Med. Biol. 37 (1992) 731.

[8] J.J. Moreno-Cantú, C.J. Thompson, R.J. Zattore, IEEE Trans. Med. Imag. MI-17 (1998) 979.

[9] N. Tomic, C.J. Thompson, Investigation of the block effect on spatial resolution in PET detectors. CD_ROM Record of the IEEE Medical Imaging Conference, October 2003, Portland, OR, Paper \# M6-134.

[10] K. Kouris, E.S. Garnett, G.T. Herman, J. Comput. Assist. Tomogr. 5 (1981) 744.

[11] Z.H. Cho, K.S. Hong, J.B. Ra, S.Y. Lee, IEEE Trans. Nucl. Sci. NS-28 (1981) 94.

[12] G. Herman, Technical Report No. MIPG2, Department of Computer Science, State University of New York Buffalo, 1978.

[13] A.C. Evans, C.J. Thompson, S. Marrett, E. Meyer, M. Mazza, IEEE Trans. Med. Imag. MI-10 (1991) 90.

[14] National Electrical Manufacturers Association, NEMA Standards Publication NU-2 2001: Performance Measurements of Positron Emission Tomographs, National Electrical Manufacturers Association, Rosslyn, VA, USA, 2001.

[15] C. Knoess, S. Siegel, A. Smith, et al., Eur. J. Nucl. Med. 30 (2001) 737.

[16] A. Chatziioannou, R.W. Silverman, K. Meadows, T.H. Farquhar, S.R. Cherry, IEEE Trans. Nucl. Sci. NS-47 (2000) 422.

[17] K. Wienhard, M. Schmand, M.E. Casey, et al., IEEE MIC (2000) Conference Record 3.5.1, Lyon, France.

[18] J.L. Robar, C.J. Thompson, K. Murthy, R. Clancy, A.M. Bergman, Nucl. Instr. and Meth. A 392 (1997) 402. 